NASA CONTRACTOR REPOR T

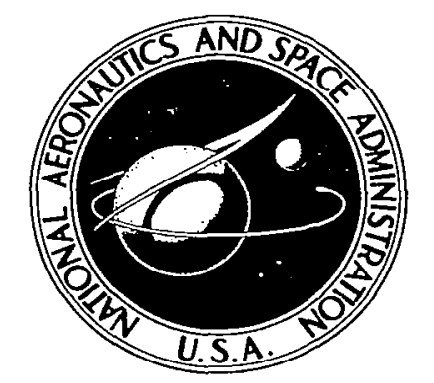

NASA CR - 9 $A: l$

$a$
0
1
0
4
$\vdots$
$\vdots$
2

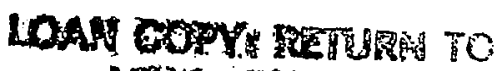

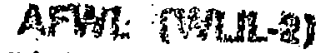

KIRTLAND AFF MANX

\title{
MAGNETOHYDRODYNAMIC FLOWS OVER A ROTATING DISK
}

by Hsien-Ping Pao

Prepared by

THE CATHOLIC UNIVERSITY OF AMERICA

Washington, D. C.

for Lewis Research Center

NATIONAL AERONAUTICS AND SPACE ADMINISTRATION - WASHINGTON, D. C. - JANUARY 1968 
NASA CR-989

\title{
MAGNETOHYDRODYNAMIC FLOWS OVER A ROTATING DISK
}

\author{
By Hsien-Ping Pao
}

Distribution of this report is provided in the interest of information exchange. Responsibility for the contents resides in the author or organization that prepared it.

Prepared under Grant No. NsG-586 by THE CATHOLIC UNIVERSITY OF AMERICA

Washington, D.C.

for Lewis Research Center

NATIONAL AERONAUTICS AND SPACE ADMINISTRATION

For sale by the Clearinghouse for Federal Scientific and Tochnical Information Springfield, Virginia 22151 - CFSTI price $\$ 3.00$ 



\section{FOREWORD}

The research described herein was conducted at the Catholic University of America under National Science Foundation Grant GP-757 and NASA Grant NsG-586 with Mr. Frank E. Rom, Nuclear Systems Division, NASA Lewis Research Center, as the Technical Manager. 


\section{SUMMARY}

The flow of an incompressible viscous electrically conducting fluid over a rotating disk is investigated. In addition, a circular magnetic field is also imposed at the disk. One physical irterest in this flow lies in the possibility of using such a magnetic field to shield a rotating body from excessive heating. Similarity assumptions lead to a reduction of the governing equations to a set of ordinary differential equations. These are integrated numerically. Some approximate solutions are also obtained.

The role of the magnetic field here is to thicken the flow boundary layer and to reduce the strength of the axial flow field. That means, the magnetic field has a diffusing and stiffening effect upon the flow field. As a consequence, the frictional moment at the disk is reduced. For sufficiently large values of the applied magnetic field, the boundary layer separates from the surface of the disk. 


\section{Introduction}

The aim of the present investigation is to gain further insight into the fundamentals of the rotating flow of a conducting fluid over a rotating disk. The corresponding non-magnetic case was

first studied by von Kármán ${ }^{\text {. Cochran }}$

of the equations. Bödewadt solved numerically the related problem of the flow produced over an infinite stationary plane in fluid which is rotating with uniform angular velocity at an infinite distance from the plane.

The problem discussed in details in this paper is the steady flow of an incompressible viscous electrically conducting fluid over a rotating disk. There is no magnetic field in the distant fluid, but in the boundary layer, there is a field in the tangential direction, generated by external means within the disk itself. One physical interest in this flow lies in the possibility of using such a field to shield a rotating body from excessive heating. Since the total pressure of a fluid element in this case constitutes of the magnetic and static pressure. The fluid pressure at the surface of the disk is then reduced by the amount of the magnetic pressure of the applied field. For a compressible fluid this would imply an equivalent reduction in density, and hence in heat transfer. The similar situation in a boundary layer flow over a magnetized

plate was discussed by Glauert

An interesting feature of the magnetic effect is that the flow boundary layer thickened and the strength of the axial flow field reduced as the strength of the magnetic field is increased. This result is completely in accord with the one obtained earlier by Pao and Long. When the applied magnetic field is sufficiently strong, the boundary layer may separate from the surface of the disk. This is in agreement with the result obtained by Galvert ${ }^{(4)}$ in a similar situation. Another prevailing feature is that the flow and the magnetic fields are diffused farther out as the kinematic viscosity or the magnetic diffusivity is increased. 
2. Formulation of the Problem

Consider an incompressible; viscous, electrically conducting fluid in axisymmetric steady (5)

motion. The governing equations in cylindrical coordinates are

$$
\begin{aligned}
& u \frac{\partial u}{\partial r}+w \frac{\partial u}{\partial z}-\frac{v^{2}}{r}=-\frac{\partial P}{\partial r}+f \frac{\partial f}{\partial r}+h \frac{\partial f}{\partial z}-\frac{g^{2}}{r}+\nu\left(\nabla^{2} u-\frac{u}{r^{2}}\right), \\
& u\left(\frac{\partial v}{\partial r}+\frac{v}{r}\right)+w \frac{\partial v}{\partial z}=f\left(\frac{\partial g}{\partial r}+\frac{g}{r}\right)+h \frac{\partial g}{\partial z}+\nu\left(\nabla^{2} v-\frac{v}{r^{2}}\right), \\
& u \frac{\partial w}{\partial r}+w \frac{\partial w}{\partial z}=-\frac{\partial P}{\partial z}+f \frac{\partial h}{\partial r}+h \frac{\partial h}{\partial z}+\nu \nabla^{2} w \\
& \frac{\partial(r u)}{\partial r}+\frac{\partial(r w)}{\partial z}=0 \\
& \frac{\partial(r f)}{\partial r}+\frac{\partial(r h)}{\partial z}=0 \\
& \frac{\partial}{\partial r}(v f-u g)+\frac{\partial}{\partial z}(v h-w g)+\eta\left(\nabla^{2} g-\frac{g}{r^{2}}\right)=0, \\
& (u h-w f)-\eta\left(\frac{\partial h}{\partial r}-\frac{\partial f}{\partial z}\right)=0
\end{aligned}
$$

with

$$
\nabla^{2}=\frac{\partial^{2}}{\partial r^{2}}+\frac{1}{r} \frac{\partial}{\partial r}+\frac{\partial^{2}}{\partial z^{2}}
$$

where $(u, v, w)$ and $(f, g, h)$ denote the components of velocity and of normalized magnetic field strength in the direction of the $(r, \theta, z)$ coordinate lines; $\nu$ is the kinematic viscosity, and $\quad \eta=1 /(\mu \sigma)$ is the magnetic diffusivity, with $\mu$ the magnetic permeability and $\sigma$ the electrical conductivity. We have written $(f, g, h)=\left(f_{1}, g_{1}, h_{1}\right) \sqrt{\mu / \rho} \quad$ and $\quad P=\left(f^{2}+g^{2}+h^{2}\right) / 2+p / \rho+X$ 
where $\left(f_{1}, g_{1}, h_{1}\right)$ are the components of the magnetic field, $p$ is fluid pressure, $f$ is uniform density and $X$ is the potential per unit mass of the conservative body forces. It is also assumed that the net charge density is zero, and that $\nu, \sigma$, and $\mu$ are constant.

Equations (4) and (5) can be integrated by introducing two scalar functions, $\psi(r, z)$ and $\Lambda\left(r_{8} z\right)$, such that

$$
\begin{array}{ll}
u=-\frac{1}{r} \frac{\partial \psi}{\partial z}, & w=\frac{1}{r} \frac{\partial \psi}{\partial r} \\
f=-\frac{1}{r} \frac{\partial \Lambda}{\partial z}, & h=\frac{1}{r} \frac{\partial \Lambda}{\partial r}
\end{array}
$$

We now seek a similarity solution of the following form:

$$
\left.\begin{array}{rlrl}
\psi=-(\nu \omega)^{\frac{1}{2}} r^{2} m(\zeta), & \Lambda=-(\nu \omega)^{\frac{1}{2}} r^{2} n(\zeta), \\
v=r \omega G(\zeta), & g=r \Omega M(\zeta), \\
P=\frac{\lambda}{2} \omega^{2} r^{2}+\nu \omega S(\zeta), & \zeta=z(\omega / \nu)^{\frac{1}{2}},
\end{array}\right\}
$$

where $\omega$ is the characteristic angular speed of the swirling velocity field and $\Omega$ is the corresponding characteristic angular magnetic field strength. It follows that, by virtue of (8) and (9)

$$
\begin{aligned}
& u=\omega r m^{\prime}, \quad w=-2(\nu \omega)^{\frac{1}{2}} m, \\
& f=\omega r n^{\prime}, \quad h=-2(\nu \omega)^{\frac{1}{2}} n,
\end{aligned}
$$


where the prinies indicate differentiation with respect to $\zeta$. Substifution of (10) - (12) into (1) $-(3),(6)$ and (7) leads to the ordinary differential equations

$$
\begin{aligned}
& \left(m^{\prime 2}-2 m m^{\prime \prime}-G^{2}\right)-\left(n^{\prime 2}-2 n n^{\prime \prime}-\beta^{2} M^{2}\right)-m^{\prime \prime \prime}+\lambda=0, \\
& 2\left(m G^{\prime}-m^{\prime} G\right)-2 \beta\left(n M^{\prime}-n^{\prime} M\right)+G^{\prime \prime}=0, \\
& S^{\prime}+4\left(m m^{\prime}-n n^{\prime}\right)+2 m^{\prime \prime}=0, \\
& \beta M^{\prime \prime}+2 \alpha\left(\beta M^{\prime} m-G n\right)=0, \\
& n^{\prime \prime}+2 \alpha\left(m n^{\prime}-m^{\prime} n\right)=0,
\end{aligned}
$$

where $\quad \alpha=\nu / \eta \quad$ is the magnetic Prandtl number and $\beta=\Omega / \omega$

Equation (15) can be integrated once

$$
S+2\left(m^{2}+m^{\prime}-n^{2}\right)=C
$$

where $C$ is a constant of integration. Hence the solution of equations (13) - (15), (17) and (18) with appropriate boundary conditions will yield a magnetohydrodynamic rotating flow over a rotating or a stationary disk which satisfies the exact magnetohydrodynamic NavierStokes equations and the equations of magnetic induction. Since a general problem of this type involves quite a few parameters, we will only consider a rather simplified case in details in the following section. 


\section{Rotating Disk and Circular Magnetic Field}

Let us consider the flow over a rotating disk with a uniform angular velocity $\omega$ in

a fluid otherwise at rest. In addition, an axial electric current of uniform current density

$J_{0}$ is imposed at the disk. Equivalently, the tangential magnetic field component is imposed at the disk which reads

$$
g=\Omega r \quad \text { with } \quad \Omega=\sqrt{\frac{\mu}{\rho}} \frac{J_{0}}{2}=\text { const. }
$$

and $f$ and $h$ are absent. The boundary conditions now assume the following form:

$$
\begin{array}{ll}
\zeta=0: & m=0, m^{\prime}=0, G=1, \quad M=1, \quad S=0, \\
S=\infty: & G=0, \quad M=0, \quad m^{\prime}=0 .
\end{array}
$$

It is noted that conditions (20) correspond to zero rotation and zero current density at $z=\infty$. If a uniform rotation and a uniform axial current density are maintained at the distant fluid, conditions (20) for $G$ and $M$ must be replaced by

$$
\zeta=\infty: \quad G=a, \quad M=b,
$$

where $a$ and $b$ are constants. The governing equations, with $n=0$, become

$$
\begin{gathered}
m^{\prime 2}-2 m m^{\prime \prime}-G^{2}+\beta^{2} M^{2}-m^{\prime \prime \prime}+\lambda=0, \\
2\left(m G^{\prime}-m^{\prime} G\right)+G^{\prime \prime}=0, \\
M^{\prime \prime}+2 \alpha m M^{\prime}=0,
\end{gathered}
$$




$$
S+2\left(m^{2}+m^{\prime}\right)=0
$$

The constant of integration in (24) vanishes because $S=m=m^{\prime}=0$ at $\zeta=0$. The constant $\lambda$ can be determined by the boundary conditions at $5=\infty: G=0, M=0, m^{\prime}=0$, together with the assumption that $\mathrm{m}^{\prime \prime}=\mathrm{m}^{\prime \prime \prime}=0$. Thus it follows from (21) that $\lambda=0$. Equation (24) is essentially an algebraic equation for $S$. Since, once $m$ is found from $(21)-(23), S$ is then known by (24). We will, therefore, consider equations (21) - (23) only. The three governing equations (21) - (23) are of seventh order altogether, hence, the seven boundary conditions in (19) and (20) are sufficient to make the problem a determinate one.

(a) Approximate Solution

An approximate solution following the Fettis method can be obtained readily. This approximate solution reveals the essential features of the flow and magnetic fields very clearly. Besides, it is very useful to the starting of the numerical integration in the next section.

The asymptotic solution is: $m=\frac{A}{2}, G=0, M=0$, and complete solution is assumed to be of the form:

$$
\begin{aligned}
& m=\frac{A}{2}+\epsilon m_{1}+\epsilon^{2} m_{2}+\epsilon^{3} m_{3}+\ldots \\
& G=\epsilon G_{1}+\epsilon^{2} G_{2}+\epsilon^{3} G_{3}+\cdots \\
& M=\epsilon M_{1}+\epsilon^{2} M_{2}+\epsilon^{3} M_{3}+\cdots
\end{aligned}
$$

where $\epsilon$ is a parameter whose exact significance will be brought out later, and $A=\lim _{\zeta \rightarrow \infty} 2 m(\zeta)$ is an as yet unspecified constant. Inserting the series (25) into equations (21)-(23), and equating like powers of $\epsilon$, the following succession of linear equations is obtained:

$$
\begin{aligned}
& m_{1}^{\prime \prime \prime}+A m_{1}^{\prime \prime}=0, \\
& G_{1}^{\prime \prime}+A G_{1}^{\prime}=0,
\end{aligned}
$$




$$
\begin{aligned}
M_{1}^{\prime \prime}+\alpha A M_{1}^{\prime}=0, \\
m_{k}^{\prime \prime}+A m_{k}^{\prime \prime}=\sum_{i=1}^{k-1} m_{i}^{\prime} m_{k-i}^{\prime}-2 m_{i} m_{k-i}^{\prime \prime}-G_{i} G_{k-i}+\beta^{2} M_{i} M_{k-i}, \\
G_{k}^{\prime \prime}+A G_{k}^{\prime}=\sum_{i=1}^{k-1} 2\left(m_{i}^{\prime} G_{k-i}-m_{i} G_{k-i}^{\prime}\right), \\
M_{k}^{\prime \prime}+\alpha A M_{k}^{\prime}=-\sum_{i=1}^{k-1} 2 \alpha m_{i} M_{k-i}^{\prime}, \\
k=2,3,4, \cdots .
\end{aligned}
$$

The conditions (20) at infinity will be satisfied if

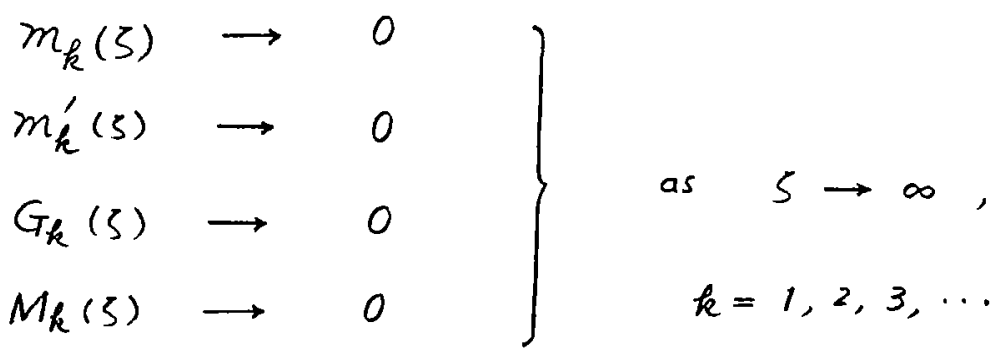

while the conditions (19) at $\zeta=0$ will be met if

$$
\begin{aligned}
& m_{1}^{\prime}(0)=0, \quad G_{1}(0)=1, \quad M_{1}(0)=1, \\
& m_{k}^{\prime}(0)=0, \quad G_{k}(0)=0, M_{k}(0)=0, \quad k=2,3,4, \cdots,
\end{aligned}
$$

and if $\epsilon=1$. However, the condition $m(0)=0$ will not be satisfied unless the constant $A$ had been so chosen that

$$
\frac{A}{2}+m_{1}(0)+m_{2}(0)+\cdots=0
$$

Equation (29) will be a transcendental equation for the determination of the unknown constant $A$.

The solution to the above system is listed as follows: 


$$
\begin{aligned}
& m_{0}=A / 2, \\
& m_{1}=0 \text {, } \\
& m_{2}=\left(a_{1} e^{-A S}+a_{2} e^{-2 A S}+a_{3} e^{-2 \alpha A S}\right) / A^{3} \text {, } \\
& m_{3}=0 \text {, } \\
& m_{4}=\left[a_{4} e^{-A S}+a_{5} e^{-2 A S}+a_{6} e^{-3 A S}+a_{7} e^{-4 A S}+a_{8} e^{-2 \alpha A S}\right. \\
& \left.+a_{q} e^{-(2 \alpha+1) A S}+a_{10} e^{-(2 \alpha+2) A S}+a_{11} e^{-4 \alpha A S}\right] / A^{7}, \\
& m_{5}=0 \\
& G_{1}=e^{-\Lambda S}, \\
& G_{2}=0, \\
& G_{3}=\left[b_{1} e^{-A S}+b_{2} e^{-3 A S}+b_{3} e^{-(2 \alpha+1) A S}\right] / A^{4}, \\
& G_{4}=0 \text {, } \\
& M_{1}=e^{-\alpha A S}, \\
& M_{2}=0 \text {, } \\
& M_{3}=\left[c_{1} e^{-\alpha A S}+c_{2} e^{-(\alpha+1) A S}+c_{3} e^{-(\alpha+2) A S}+c_{4} e^{-3 \alpha A S}\right] / A^{4} \\
& M_{4}=0
\end{aligned}
$$

where

$$
\begin{aligned}
& a_{1}=\frac{1}{2}+\frac{\beta^{2}}{2 \alpha(1-2 \alpha)}, \quad a_{2}=\frac{1}{4}, \quad a_{3}=\frac{\beta^{2}}{4 \alpha^{2}(1-2 \alpha)}, \\
& b_{1}=\frac{1}{12}-\frac{\beta^{2}}{4 \alpha^{3}(2 \alpha+1)}, \quad b_{2}=-\frac{1}{12}, \quad b_{3}=\frac{\beta^{2}}{4 \alpha^{3}(2 \alpha+1)}, \\
& c_{1}=\alpha^{2}\left[\frac{1}{\alpha+1}-\frac{1}{4(\alpha+2)}\right]-\frac{\beta^{2}}{12 \alpha^{2}(1-2 \alpha)}\left(1-\frac{12 \alpha^{3}}{\alpha+1}\right), \\
& c_{2}=-\frac{\alpha^{2}}{\alpha+1}\left[1+\frac{\beta^{2}}{\alpha(1-2 \alpha)}\right], \quad c_{3}=\frac{\alpha^{2}}{4(\alpha+2)}, \quad c_{4}=\frac{\beta^{2}}{12 \alpha^{2}(1-2 \alpha)}, \\
& a_{4}=-\left[\frac{1}{144}+\frac{a_{1}}{4}+2 a_{5}+2 \alpha a_{8}+(2 \alpha+1) a_{9}+2(\alpha+1) a_{10}+4 \alpha a_{11}\right], \\
& a_{5}=\frac{1}{4}\left(a_{1}^{2}+2 b_{1}\right), \quad a_{6}=\frac{a_{1}}{12}, \quad a_{7}=\frac{1}{576}, \quad a_{8}=\frac{\beta^{2} c_{1}}{2 \alpha^{2}(1-2 \alpha)}, \\
& a_{9}=\frac{a_{1} a_{3}\left(4 \alpha^{2}-2 \alpha+1\right)-\beta^{2} c_{2}}{\alpha(2 \alpha+1)^{2}}, \quad a_{10}-\frac{a_{3}\left(\alpha^{2}-\alpha+1\right)+b_{3}-\beta^{2} c_{3}}{2(\alpha+1)^{2}(2 \alpha+1)}, \quad a_{11}=\frac{2 \alpha^{2} a_{3}^{2}-\beta^{2} c_{4}}{8 \alpha^{2}(4 \alpha-1)} .
\end{aligned}
$$

$-9-$ 
It is noted that for $\alpha=\frac{1}{2}$, the l'Hospital's rule of taking limits has to be used. The above approximate solution coverges quite rapidly provided that $\beta^{2}$ is small or $\alpha$ is large.: $A$ comparison of the 4 - term approximate solution with the "exact" numerical solution reveals that they are practically indistinguishable for the range $\beta^{2} / \alpha \leq 0.1$. When $\beta^{2}$ becomes larger and $\alpha$ smaller, the convergence of the approximate series solution becomes slower. The series solution fail to converge when $\alpha \leqslant 1$ and $\beta^{2} \geqslant 1$. The above feature is also indicated by the numerical solution. Let us consider a special case $\alpha=1$ and $\beta^{2}=1$. It can be verified readily that the solution is

$$
m=\frac{a^{*}}{2}, G=e^{-a^{*} \zeta}, \quad M=e^{-a^{*} \zeta},
$$

which corresponds to a suction at the rotating disk with a strength $a^{*}(\nu(\omega))^{\frac{1}{2}}$. In the limit as the suction vanishes, we hove $G \rightarrow 1$ and $M \rightarrow 1$. That is, the fluid and the disk undergo a rigid body rotation. This is a common feature of the magnetohydrodynamic flow in an aligned field $(\underline{5}, \underline{7}, \underline{8}, \underline{9})$

When $\alpha \leq 1$ and $\beta>1$, then it appears that no steady similarity solution is possible unless there is suction acting, which prevents the boundary layer from loaving the disk. This is in accord (6) with the conclusion reached by Fettis , and Rogers and Lance ${ }^{-}$in a similar situation. It is, therefore, anticipated that for each valve of the suction parameter $a^{*}$ there are critical values of the pair $(\alpha, \beta)$ corresponding to which no steady flow is possible.

(b) Numerical Integration

The differential equations requiring numerical methods for their solution are equations (21) $-(23)$. Solutions of these equations are required which satisfy the boundary conditions (19) and $(20)$. 
The asymptotic solution of the obove system is

$$
\left.\begin{array}{l}
m \rightarrow \frac{A}{2} \\
G \rightarrow b e^{-A S} \\
M \rightarrow c e^{-\alpha A S}
\end{array}\right\} \quad \text { as } 5 \rightarrow \infty
$$

where $A, b$, and $c$ are constant. In order to start the numerical integration of the above system at $\zeta=0$, it is necessary to estimate the values of $m^{\prime \prime}(0), G^{\prime}(0)$ and $M^{\prime}(0)$. Reasonably accurate estimates can be made using the values from the approximate solution. With these values, the system was integrated from zero to $\zeta=10$. The behavior of the solution is tested by the following scheme:

$$
\left.\begin{array}{l}
G=b_{1}+b_{2} e^{-A_{1} \zeta} \\
M=c_{1}+c_{2} e^{-\alpha A_{2} \zeta} \\
m^{\prime}=d_{1}+d_{2} e^{-A_{3} \zeta}
\end{array}\right\} \quad \text { at } \zeta=9.8,9.9,10
$$

and

$$
m(10)=A / 2
$$

Three conditions were imposed:

$$
b_{1}=0, \quad c_{1}=0, \quad \text { and } \quad d_{1}=0
$$

This was the basis for the numerical integration. The conditions (35) can be satisfied by adjusting the values of $m^{\prime \prime}(0), G^{\prime}(0)$ and $M^{\prime}(0)$. The computation indicates that once the conditions (35) are satisfied, then the following relation also follows

$$
A_{1}=A_{2}=A
$$

correct to the third significant figure. Hence, the asymptotic conditions (33) are fulfilled, and the numerical solution is thus obtained. 


\section{(c) Numerical Results}

Many results have been mentioned in the previous sections, but some graphs and tables are presented here to emphasize the more important points. Figures $1-3$ show the velocity and magnetic fields for various values of $\alpha$ and $\beta$. We observe that as $\beta$ increases, the axial velocity strength decreases while the boundary layer thickness of the flow and the magnetic fields increases. Hence, an increase of the circular magnetic field strength has an effect of thickening the boundary layer and reducing the strength of the axial velocity field. Figure 4 shows the effect of the magnetic Prandtl number $\alpha$. It is observed that the boundary layer thickness of the flow and the magnetic fields decreases as $\alpha$ increases. The kinematic viscosity $\nu$ is known, from the similarity form (10), to diffuse the momentum farther out from the boundary. Hence the effect of the magnetic Prandtl number confirms that the magnetic diffusivity is also to diffuse the magnetıc field out and thus thickens the boundary layer.

The derivatives of the velocity and the magnetic fields at the disk are given in Table 1 for various values of $\alpha$ and $\beta$. From these values, some other characteristics of the flow and magnetic fields can be computed, in particular the frictional moment on the disk. Neglecting edge effects, we may write the moment of the frictional forces acting on a disk of large but finite radius $R$ as

$$
\begin{aligned}
M & =-2 \pi \int_{0}^{R} r^{2} \rho \nu\left(\frac{\partial v}{\partial z}\right)_{z} d r \\
& =-\frac{1}{2} \rho \pi R^{4}\left(\nu \omega^{3}\right)^{\frac{1}{2}} G^{\prime}(0) .
\end{aligned}
$$

Or introducing the dimensionless frictional moment coefficient, we have

$$
C_{M}=\frac{M}{\frac{1}{2} \rho\left(R(\omega)^{2} \pi R^{3}\right.}=-\frac{G^{\prime}(0)}{R_{e}^{\frac{1}{2}}}, \quad \operatorname{Re}=\frac{R^{2} \omega}{\nu} \text {. }
$$


The frictional moment coefficient, $C_{M}$, and the axial velocity strength at distance fluid, $A$, are further illustrated in Fig. 5. It is observed that $C_{M}$ and $A$ decrease as $\beta^{2}$ increases or as $\alpha$ decreases. Other interesting feature of the flow and magnetic fields is the distribution of the vorticity and the electrical current density. By virtue of $(10)-(12)$ we obtain

$$
\left.\begin{array}{l}
\xi_{r}=-\frac{\partial v}{\partial z}=-\left(\omega^{3} / \nu\right)^{\frac{1}{2}} r G^{\prime}(s) \\
\xi_{\theta}=\frac{\partial u}{\partial z}-\frac{\partial u r}{\partial r}=\left(\omega^{3} / \nu\right)^{\frac{1}{2}} r m^{\prime \prime}(s) \\
\xi_{z}=\frac{1}{r} \frac{\partial}{\partial r}(v r)=2 \omega G(s)
\end{array}\right\}
$$

and

$$
\begin{aligned}
& J_{r}=-\left(\frac{\rho}{\mu}\right)^{\frac{1}{2}} \frac{\partial g}{\partial z}=-\frac{J_{0}}{2}(\omega / \nu)^{\frac{1}{2}} r M^{\prime}(s) \\
& J_{\theta}=0 \\
& J_{z}=\left(\frac{\rho}{\mu}\right)^{\frac{1}{2}} \frac{1}{r} \frac{\partial}{\partial r}(g r)=J_{0} M(s)
\end{aligned}
$$

where $\left(\xi_{r}, \xi_{\theta}, \xi_{z}\right)$ and $\left(J_{r}, J_{\theta}, J_{z}\right)$ are the components of the vorticity and the current density respectively in the $r-, \theta-, z-$ directions. The current lines as well as the vortex lines start at the disk and spread out radially. The current density and the vorticity are zero at a great distance from the disk. Hence no current lines or vortex lines penetrate to $z=\infty$. However, in the special case when $\alpha=1, \beta^{2}=1$, the stiffening effect of the magnetic field is so strong that all current lines and vortex lines penetrate to $z=\infty$ and the current density and vorticity becomes axial and uniform everywhere. It should be noted that $J_{r}$ at the disk is a derived current iust like $\xi_{r}$ at the disk is a derived vorticity.

The total pressure $S$ can readily be obtained from equation (24). In particular, $S(0)=0$ and $S(\infty)=-A^{2} / 2$. Thus, the total pressure difference across the boundary layer is $A^{2} / 2$ which decreases as the magnetic strength increases. 
4. Rotating Flow in A Circular Magnetic Field

(3)

A magnetohydrodynamic flow of Bödewadt can be considered here. In this case, the fluid at a large distance above a stationary disk rotates at a uniform angular velocity $\omega$. In addition, an axial electric current of uniform current density Jo is imposed at $z=\infty$. Thus, the tangential magnetic field component at $z=\infty$ is

$$
g=\Omega r \quad \text {, with } \Omega=\left(\frac{\mu}{\rho}\right)^{\frac{1}{2}} \frac{J_{0}}{2}=\text { const. },
$$

and $f$ and $g$ are absent. The similarity form in $(10)-(12)$ is still valid in this case and the boundary conditions now assume the following form:

$$
\begin{aligned}
& S=0: m=0, m^{\prime}=0, G=0, M=0, S=0, \\
& S=\infty: G=1, M=1, m^{\prime}=0
\end{aligned}
$$

We have used the conditions that the disk is stationary and electrically insulated, thus no current or vorticity in the axial plane passing through the disk. If a uniform rotation and a uniform axial current density are maintained at the disk, conditions (41) for $G$ and $M$ must be replaced by

$$
S=0: G=a, M=b,
$$

where $a$ and $b$ are constants. The governing equations given in (21) - (24) remain valid, except that the constant $\lambda$ in this case is no longer zero. $\lambda$ can be determined by the boundary conditions at $\zeta=\infty: G=1, M=1, m^{\prime}=0$, together with the assumption that $m^{\prime \prime}=m^{\prime \prime \prime}=0$. Thus, it follows from (21) that $\lambda=1-\beta^{2}$. Therefore, three governing equations (21) - (23) fogether with the boundary conditions (41) and (42) constitute a determinate system. Then, the approximate solution and the numerical integration can be carried out in a similar manner. 


\section{Conclusions}

A more general problem with both the disk and the distant fluid in rotation together with more general magnetic field can be treated in a similar manner. But, so many parameters involved have prohibited us from going into details for such a general problem. Nonetheless, some of the conclusions derived from the detailed investigation of the particular case (rotating disk and circular magnetic field) might still be valid for the general problem.

For the case of rotating disk and circular magnetic field, we have the following conclusions:

(1) An increase of the magnetic field stregnth has an effect of thickening the boundary layer and reducing the strength of the axial flow field. In other words, the magnetic field has a diffusing and stiffening effect upon the flow field. As a consequence, the frictional moment at the disk is also reduced when the magnetic field is increased.

(2) The boundary layer thickness of the flow and the magnetic fields increases as the kinematic viscosity or the magnetic diffusivity increases. This confirms their respective diffusive roles.

(3) The total pressure (magnetic and static) difference across the boundary layer is reduced as the magnetic field is increased. Moreover, the fluid static pressure at the surface is further reduced by the amount of the magnetic pressure of the applied field. For a compressible fluid this would imply an equivalent deduction in density, and hence in heat transfer.

(4) In the special case when $\alpha=1, \beta^{2}=1$, the fluid and the disk undergo a rigid body rotation, thus indicating the beginning of the boundary layer separation. In the numerical integration, it is found that the computation becomes extremely sensitive to the choice of $m^{\prime \prime}(0), G^{\prime}(0)$ and $M^{\prime}(0)$ as $\frac{\beta^{2}}{\alpha}$ increases. When $\beta^{2}$ is large, the region of large gradients, or boundary layer, 
seems to have moved away from the disk and large shears are appearing at the distant fluid. That means, the boundary layer separates from the surface of the disk for sufficiently large values of the applied field strength. This confirms the importance of magnetically induced separation as an effect to be reckoned with in magnetohydrodynamics

(5) The suction at the disk prevents the boundary layer from leaving the disk. 


\section{REFERENCES}

1. T. Von Kármán, Z. angew. Math. Mech. I (1921) 244.

2. W. G. Cochran, Proc. Camb. Phil Soc. 30 (1934) 365.

3. U. T. Bödewadt, Z. angew. Math. Mech. 20 (1940) 241.

4. M. B. Glavert, J. Fluid Mech. 12 (1962) 625.

5. H.P. Pao and R. R. Long, Quart. J. Mech. Appl. Math. 19 (1966) 1.

6. H. E. Fettis, Proc. 4th Midwest Conf. on Fluid Mech., (1955) 93.

7. H.P. Greenspan and G.F. Carrier, J. Fluid Mech. $\underline{6}$ (1959) 77.

8. G. F. Carrier and H.P. Greenspan, J. Fluid Mech $Z$ (1960) 22.

9. M.B. Glavert, J. Fluid Mech. 10 (1961) 276.

10. M. H. Rogers and G. N. Lance, J. Fluid Mech 7 (1960) 617. 
Table 1. Values of $m^{\prime \prime}(0), G^{\prime}(0), M^{\prime}(0)$ and $2 m(\infty)$ for various values of $\alpha$ and $B$

\begin{tabular}{|c|c|c|c|c|c|}
\hline & B & $\mathrm{m}^{\prime \prime}(0)$ & $G^{\prime}(0)$ & $M^{\prime}(0)$ & $2 m(\infty)$ \\
\hline $\begin{array}{c}\eta \\
0 \\
11 \\
\gamma\end{array}$ & $\begin{array}{l}0 \\
.05 \\
.1 \\
.15 \\
.2 \\
.25 \\
.3\end{array}$ & $\begin{array}{l}0.51024 \\
0.50886 \\
0.50470 \\
0.49778 \\
0.48806 \\
0.47550 \\
0.46016\end{array}$ & $\begin{array}{l}-0.61592 \\
-0.61501 \\
-0.61223 \\
-0.60749 \\
-0.60054 \\
-0.59102 \\
-0.57776\end{array}$ & $\begin{array}{l}-0.26227 \\
-0.26170 \\
-0.25997 \\
-0.25696 \\
-0.25236 \\
-0.24573 \\
-0.23514\end{array}$ & $\begin{array}{l}0.886 \\
0.882 \\
0.871 \\
0.852 \\
0.823 \\
0.778 \\
0.700\end{array}$ \\
\hline $\begin{array}{l}- \\
\gamma \\
\gamma\end{array}$ & $\begin{array}{l}0 \\
.1 \\
.2 \\
.3 \\
.4 \\
.5 \\
.6 \\
.7\end{array}$ & $\begin{array}{l}0.51024 \\
0.50558 \\
0.49154 \\
0.46786 \\
0.43406 \\
0.38936 \\
0.33266 \\
0.26296\end{array}$ & $\begin{array}{l}-0.61592 \\
-0.61362 \\
-0.60657 \\
-0.59423 \\
-0.57552 \\
-0.54834 \\
-0.50822 \\
-0.44319\end{array}$ & $\begin{array}{l}-0.39625 \\
-0.39469 \\
-0.38991 \\
-0.38152 \\
-0.36872 \\
-0.34998 \\
-0.32186 \\
-0.27457\end{array}$ & $\begin{array}{l}0.886 \\
0.880 \\
0.867 \\
0.844 \\
0.809 \\
0.755 \\
0.669 \\
0.506\end{array}$ \\
\hline $\begin{array}{l}\stackrel{0}{A} \\
\gamma \\
\gamma\end{array}$ & $\begin{array}{l}0 \\
.2 \\
.4 \\
.6 \\
.8 \\
1.0 \\
1.2 \\
1.3\end{array}$ & $\begin{array}{r}0.51024 \\
0.50058 \\
0.47120 \\
0.42078 \\
0.34650 \\
0.24202 \\
0.08714 \\
-0.07124\end{array}$ & $\begin{array}{l}-0.61592 \\
-0.61378 \\
-0.60717 \\
-0.59540 \\
-0.57695 \\
-0.54816 \\
-0.49600 \\
-0.41399\end{array}$ & $\begin{array}{l}-1.13399 \\
-1.12916 \\
-1.11380 \\
-1.08638 \\
-1.04322 \\
-0.97545 \\
-0.85155 \\
-0.65578\end{array}$ & $\begin{array}{l}0.886 \\
0.884 \\
0.878 \\
0.870 \\
0.857 \\
0.838 \\
0.805 \\
0.757\end{array}$ \\
\hline
\end{tabular}



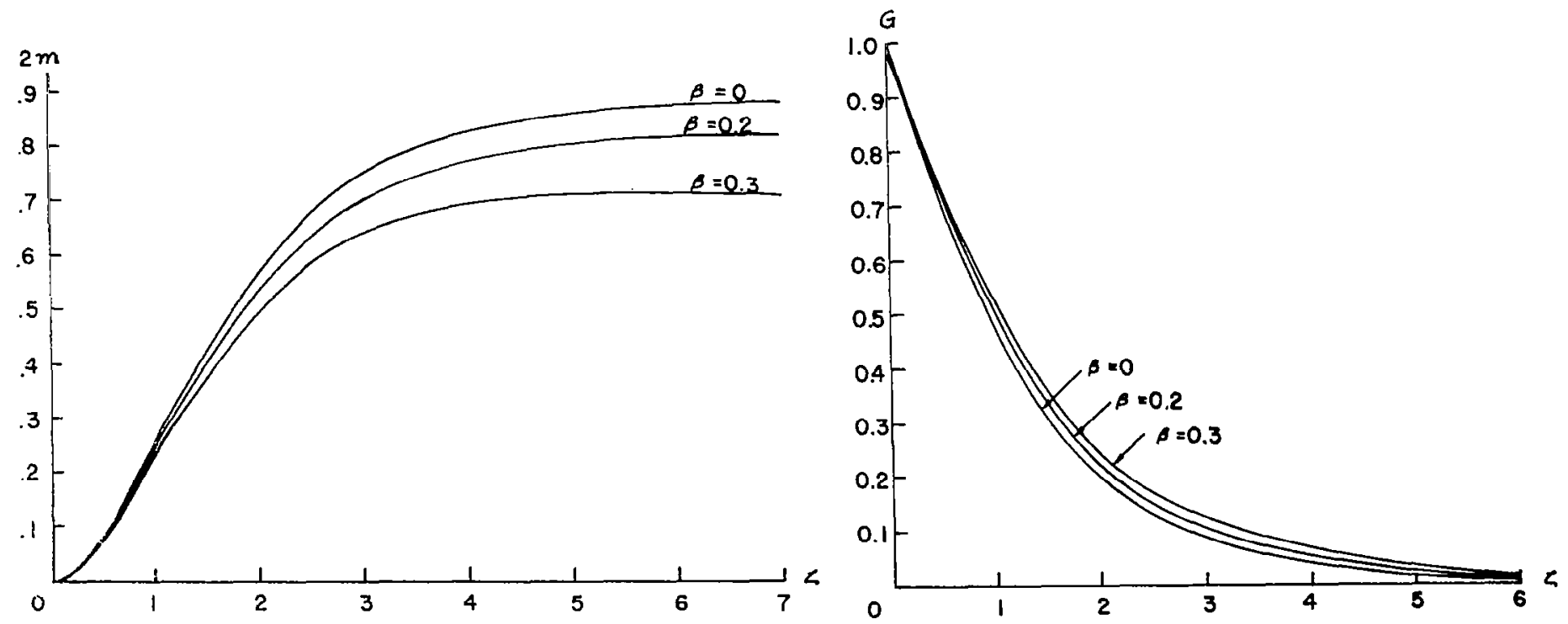

\section{(a) * eurves}
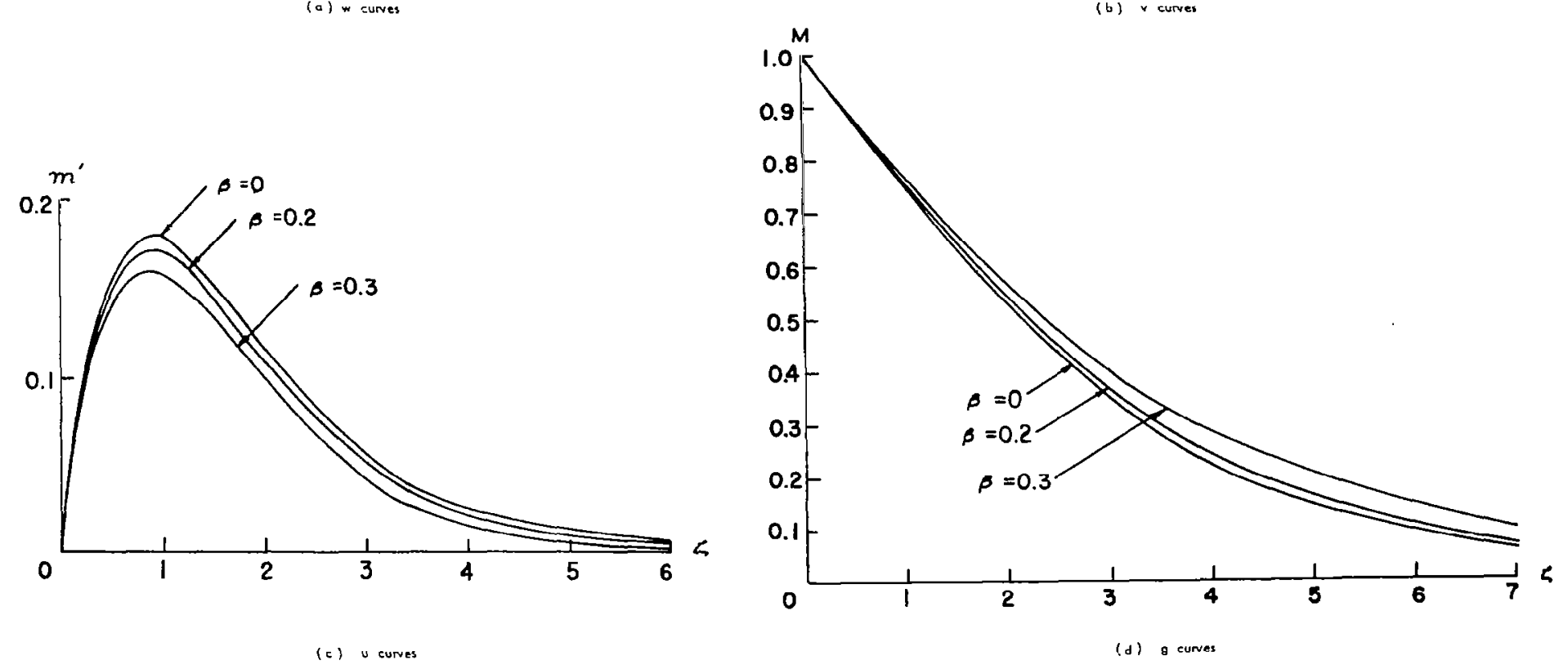


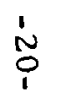
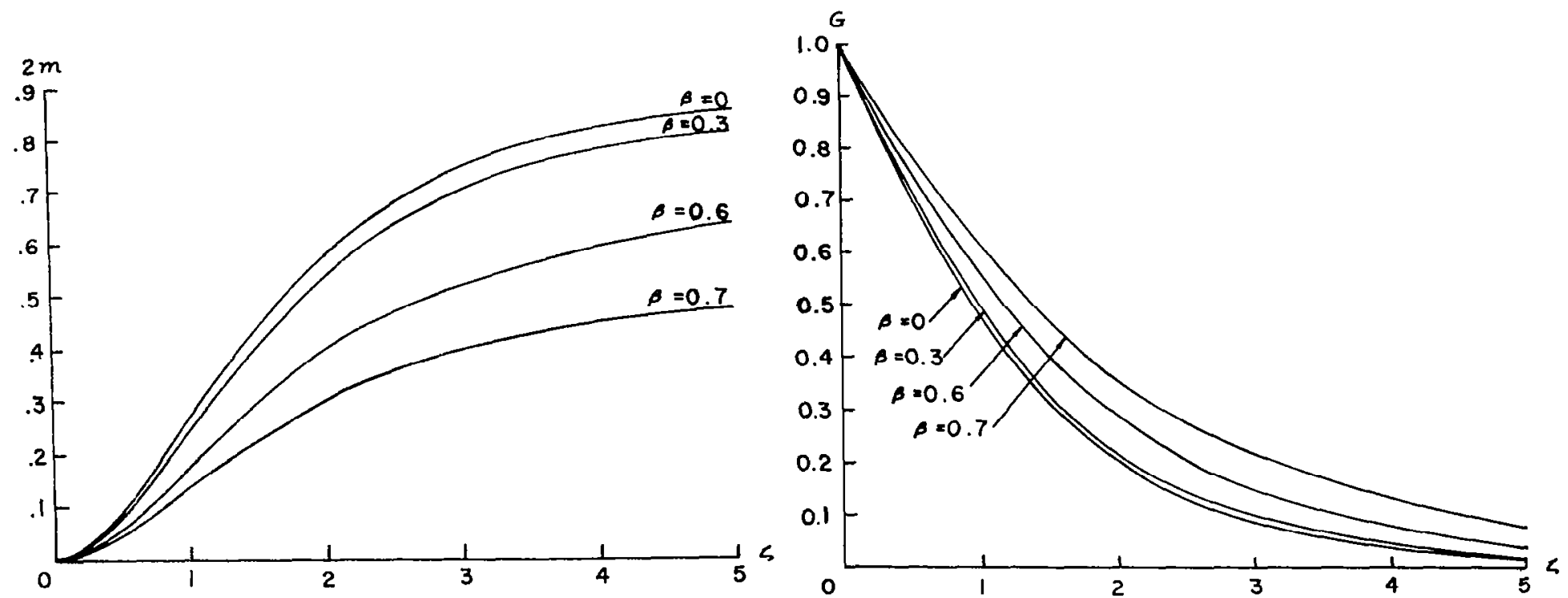

(a) w eurves
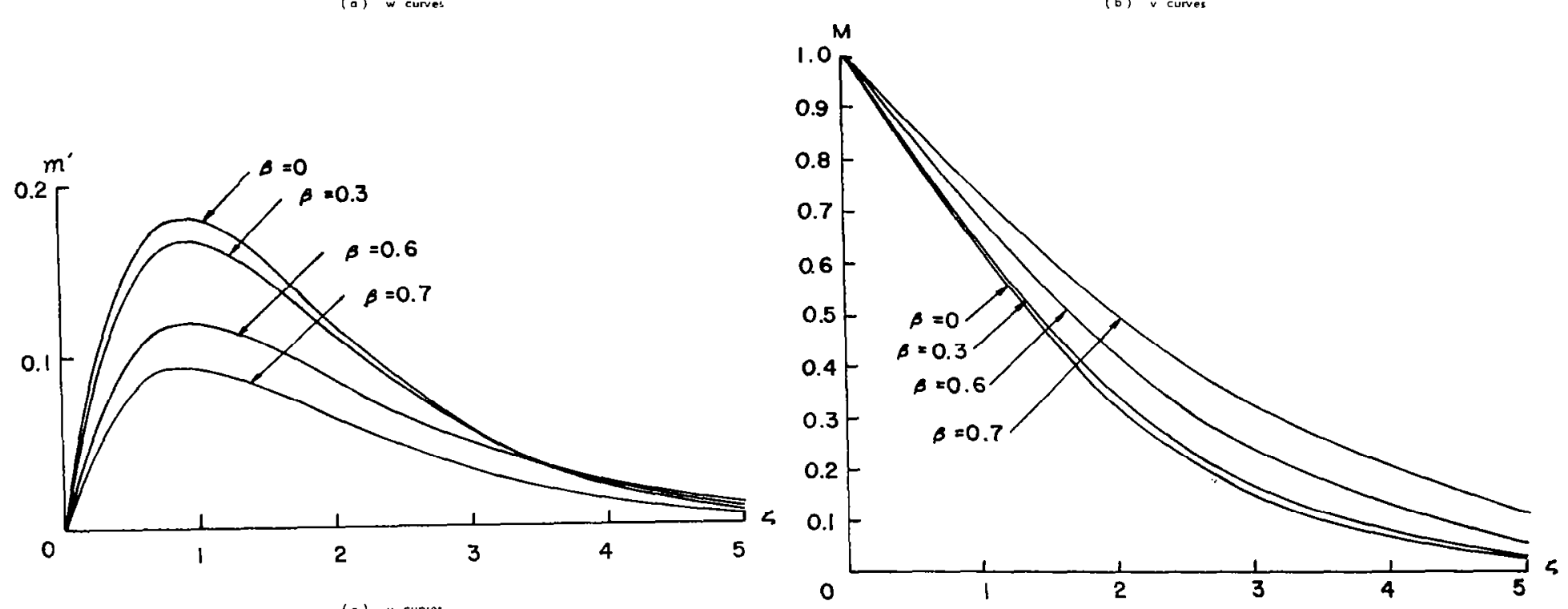

(c) ourves

(d) $g$ curves 

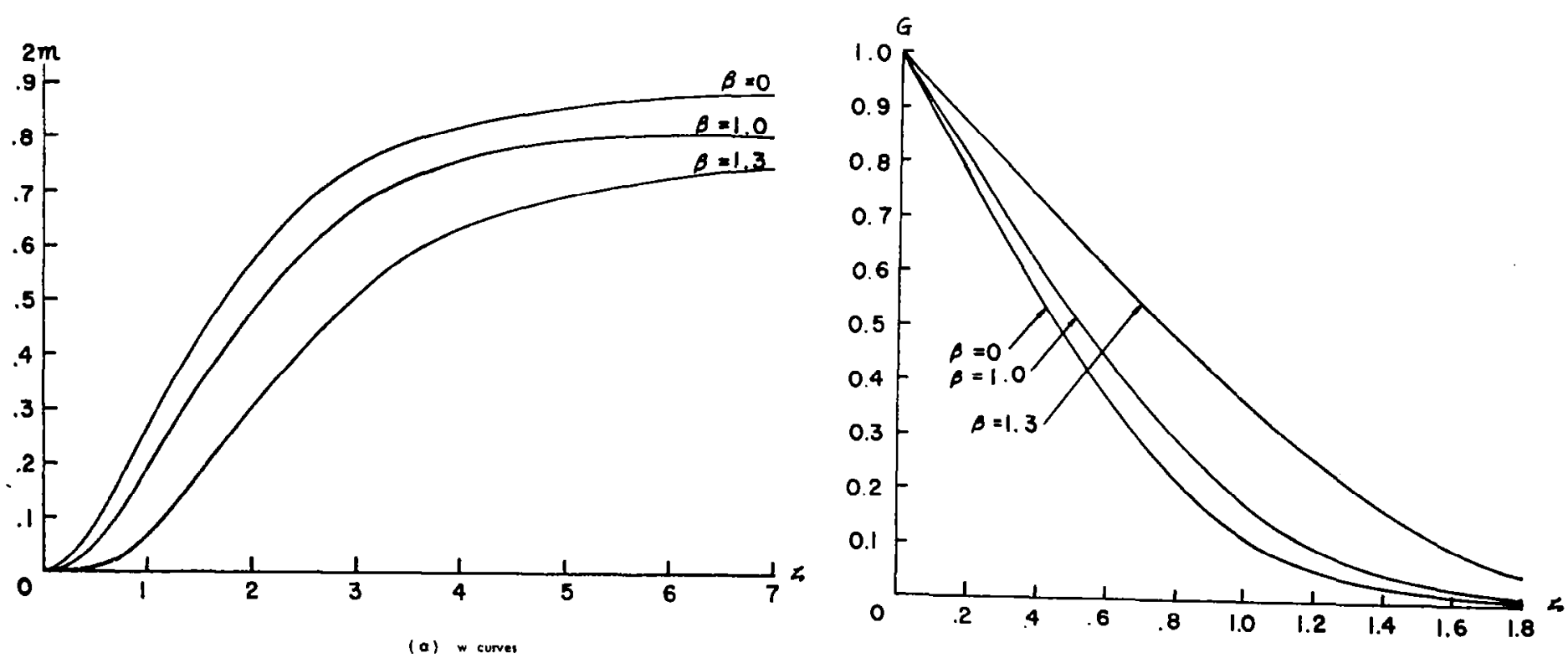

(a) w curves
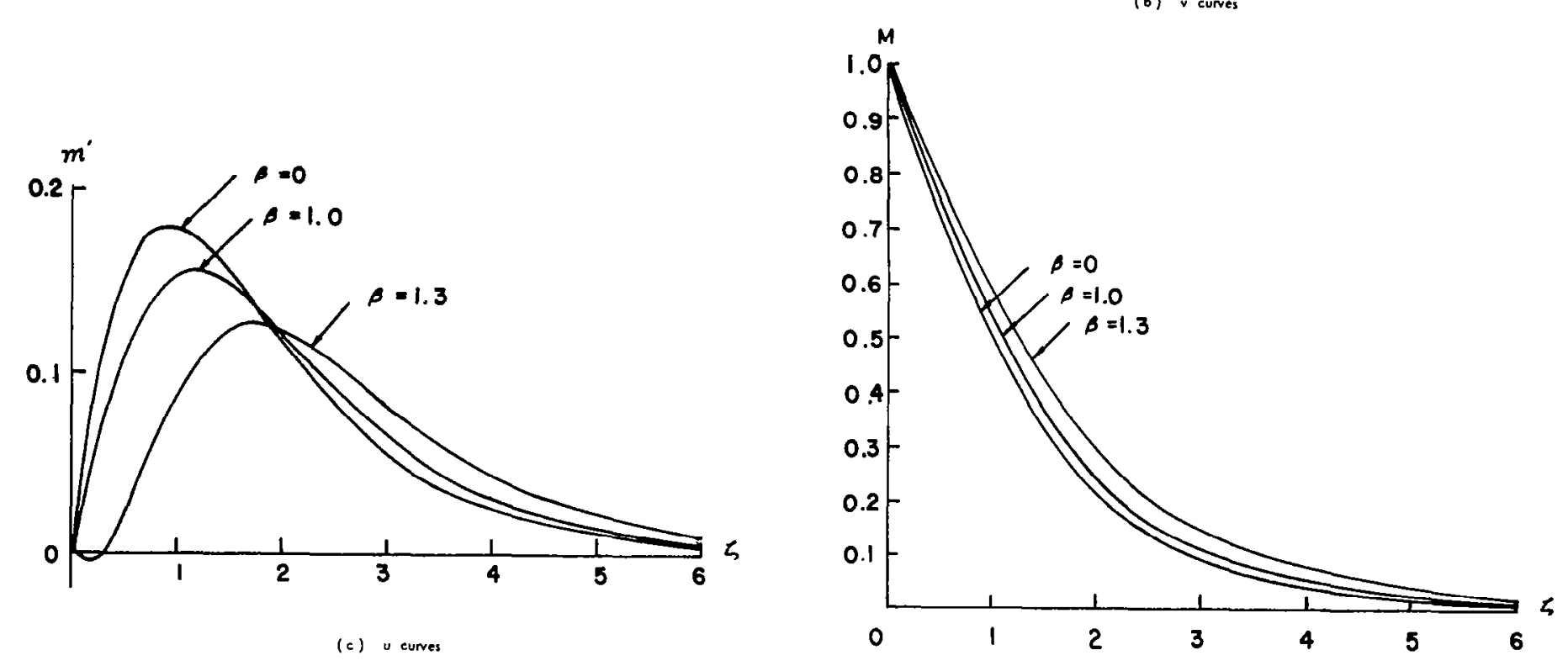

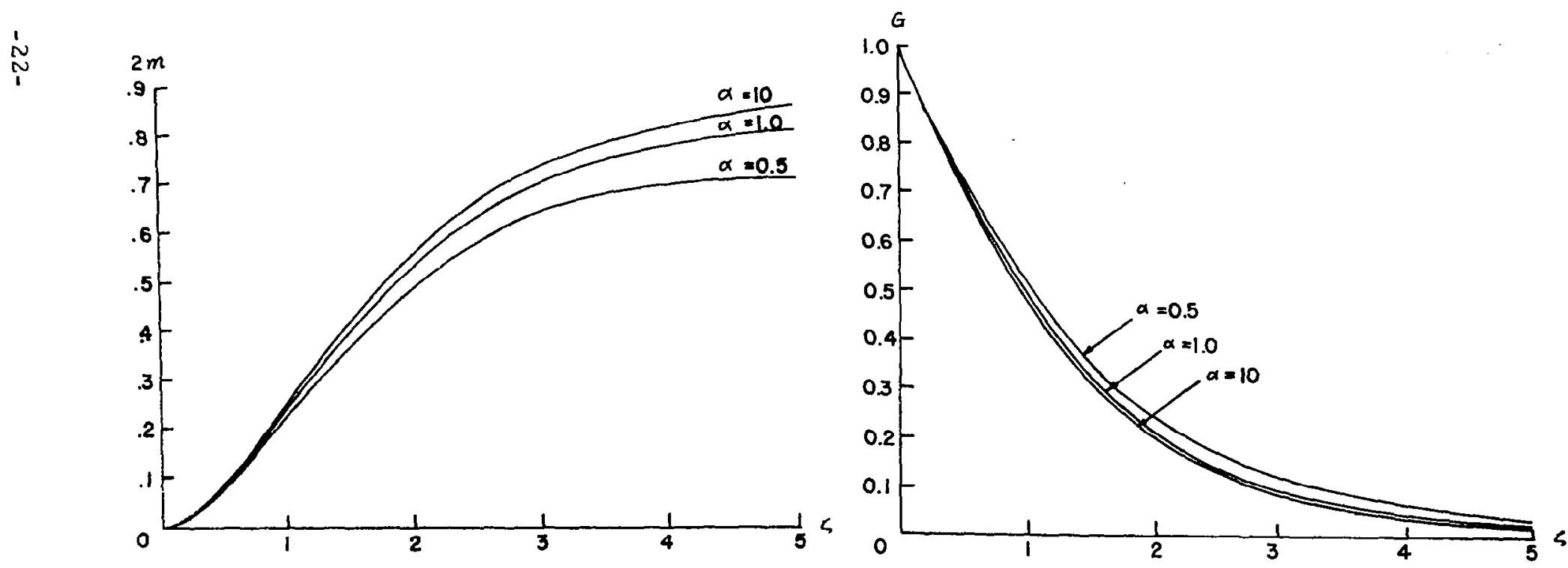

(0) w curves
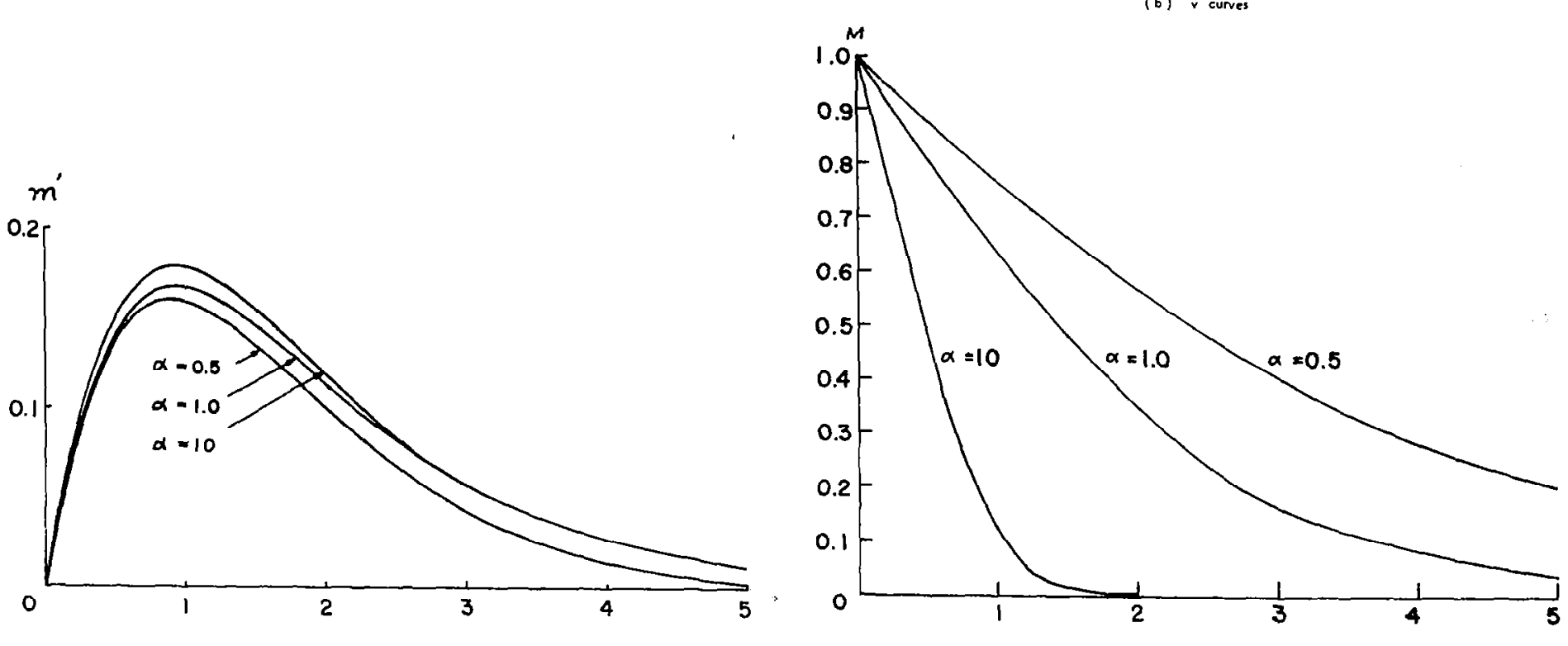

(c) $u$ eurres 


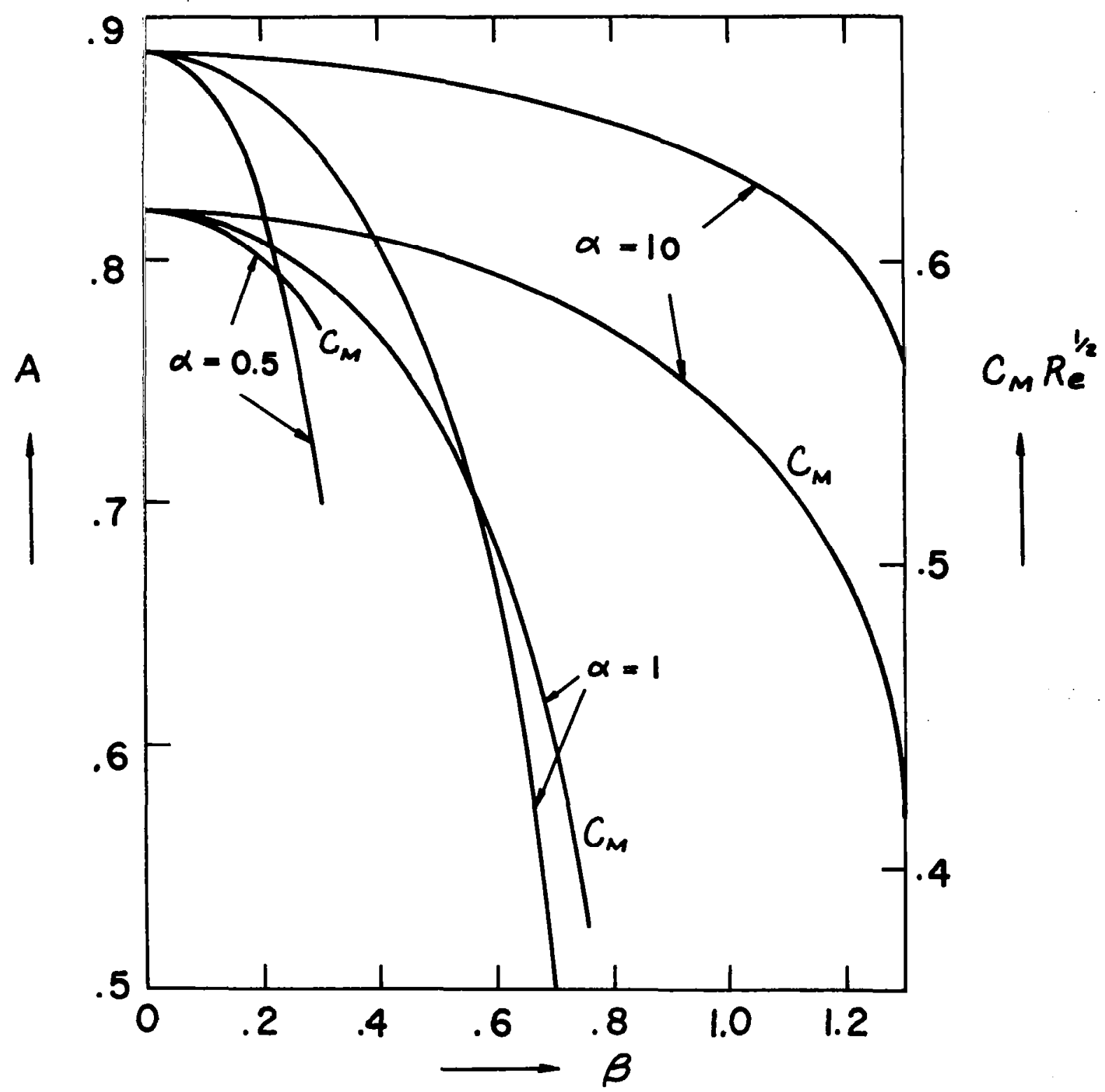

Figure 5. The frictional moment coefficient, $C_{M}$, and the axial velocity strength at the distant fluid, $A$. 